\section{Análisis de sobrevida en pacientes de edad avanzada que inician hemodiálisis crónica en Servicio de Salud Chileno}

\author{
ÁLVARO RÍOS ${ }^{1}$, PATRICIA HERRERA ${ }^{1}$, \\ ÁLVARO MORALES ${ }^{a}$, ENRIQUE REYNOLDS ${ }^{1}$, \\ MARÍA BELÉN FERNÁNDEZ ${ }^{\mathrm{b}}$, FERNANDO GONZÁLEZ ${ }^{2}$
}

\section{Survival of older patients starting hemodialysis in Chile}

Background: The proportion of older people with end stage renal disease is increasing. Their prognosis is characterized by a high mortality and poor quality of life. Aim: To analyze the survival of patients starting chronic hemodialysis (CHD) according to their age. Material and Methods: Patients admitted to CHD in the East Metropolitan Health Service of Santiago in a 2-year period were analyzed. Four age groups were created, separating patients older than 70 years in a special group. Results: During the study period, 459 patients were admitted to CHD and were followed for an average of 27 months. The frequency of cardiovascular comorbidity, cancer, and chronic renal disease of unknown cause (attributed to nephrosclerosis) increased along with age. Mortality was higher at older ages. There was a significant association between starting CHD with a catheter, Charlson comorbidity index and increasing age with mortality. For those aged over 80 years, mortality at three months and one year was 25 and 43\% respectively. Conclusions: Age, Charlson index and vascular access are predictors of mortality in older adults entering hemodialysis. This study suggests the importance of considering comorbidities, assessment by specialists and creating an arteriovenous fistula in this age group.

(Rev Med Chile 2016; 144: 697-703)

Key words: Frail Elderly; Kidney Failure, Chronic; Renal Dialysis.

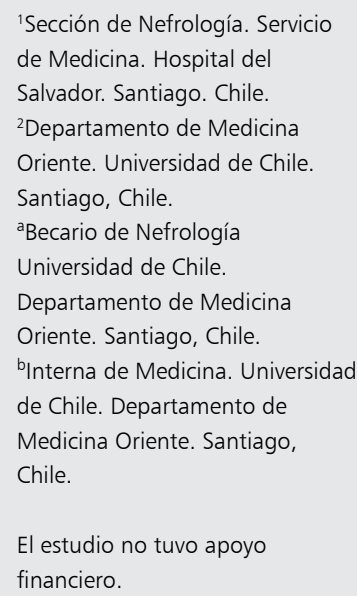

Recibido el 13 de noviembre de 2015, aceptado el 6 de abril de 2016.

Correspondencia a: Dr. Álvaro Ríos Sandoval Hospital del Salvador. Departamento de Medicina. Av. Salvador 364. Providencia. Santiago, Chile alvarorios82@gmail.com
E 1 aumento de la esperanza de vida y el envejecimiento progresivo de la población a nivel mundial supone nuevos desafíos para los sistemas de salud en múltiples aspectos, en especial en lo que se refiere a salud cardiovascular y renal ${ }^{1}$. Debido a que la prevalencia de enfermedad renal crónica aumenta con la edad, el número de personas de edad avanzada que presentan enfermedad renal crónica terminal (ERCT) está aumentando de manera alarmante ${ }^{2}$.

A nivel internacional, datos de la United States Renal Data System (USRDS), revelan que el grupo de pacientes en hemodiálisis crónica (HDC) que más ha crecido entre los años 2002 y 2012, son los mayores de 60 y de 80 años, con un aumento de $62 \%$ y $72,5 \%$ respectivamente ${ }^{3}$. En Chile, durante el mismo período la prevalencia de este grupo de pacientes ha aumentado aún más, en torno a $96 \%$ para los mayores de 60 años, y 300\% los de más de $80^{4}$.

En nuestro país, existe un aumento progresivo de la esperanza de vida de la población que se ha acompañado de un cambio en la distribución de la tasa de mortalidad, concentrándose en el grupo de mayores de 75 años. En este grupo, las enfermedades cardiovasculares y el cáncer son las principales causas de muerte ${ }^{5}$. La Encuesta Nacional de Salud (2009-10) ${ }^{6}$ muestra que los 
mayores de 65 años tienen una prevalencia de función renal disminuida (definida como $\mathrm{VFGe}<$ $60 / \mathrm{ml} / \mathrm{min} / 1,73 \mathrm{mt}^{2}$ por la fórmula MDRD-4) de $10,5 \%$. Por otro lado, el grupo mayor de 80 años tiene una alta prevalencia de sobrepeso $(26 \%)$, hipertensión arterial $(85 \%)$, dislipidemia $(63 \%)$ y alto riesgo cardiovascular $(52 \%)^{7}$; todos estos datos hacen suponer que existe un elevado riesgo de ERCT en esta población de pacientes. El Registro de Diálisis Chileno del año 2013 reveló que 37,7\% de los ingresos anuales corresponde a mayores de 65 años, además $64,9 \%$ de los pacientes fallecidos ese año fueron mayores de 70 años ${ }^{4}$.

La importancia de considerar la ERCT como un problema de salud pública en los pacientes de edad avanzada, radica no sólo en el alto costo económico de la terapia de sustitución renal, sino que también en su resultado en este grupo etario. La menor expectativa de vida y la mayor prevalencia de comorbilidades afectan negativamente la sobrevida durante el tratamiento de sustitución renal. Datos internacionales muestran que la mortalidad anual, en los mayores de 80 años, que inician diálisis, es de $54 \%$, y en $20 \%$ esta ocurre durante los primeros 3 meses, y además la mortalidad precoz se acompaña además de un importante deterioro funcional ${ }^{8}$. Pese a la relevancia de estos datos, en Chile no existen estudios prospectivos que evalúen la sobrevida de los pacientes de edad avanzada con ERCT en tratamiento con HDC.

En este estudio se comparara la sobrevida actuarial de pacientes que inician HDC en nuestro Servicio de Salud, según la edad de ingreso a la terapia, con énfasis en la población mayor o igual de 70 años. Se analiza además la relación entre la causa de ERCT, el acceso vascular y las comorbilidades con la mortalidad en cada grupo.

\section{Materiales y Métodos}

Se incluyeron a todos los pacientes mayores o igual a 18 años de edad que ingresaron al programa nacional de Hemodiálisis Crónica (HDC) en el Servicio de Salud Metropolitano Oriente (SSMO), entre el 01 de enero de 2010 y el 31 de diciembre de 2012, y que hayan sido dados de alta del hospital a un régimen de diálisis crónica ambulatoria. Se obtuvo la información de la fecha de ingreso a HDC, diagnóstico de ERCT, acceso vascular, y comorbilidades del Registro de Ingreso a HDC del
SSMO que lleva el Hospital del Salvador. Como comorbilidades se consideraron la presencia de hipertensión arterial (HTA), diabetes mellitus (DM), cáncer, y enfermedad cardiovascular (ECV) definida como la presencia de infarto al miocardio, accidente cerebrovascular, enfermedad vascular periférica o de la aorta. Para todos los pacientes se calculo el Índice de Comorbilidad de Charlson (IC), que ha sido validado en población general ${ }^{9}$, y también en población con ERCT ${ }^{10}$. La edad de ingreso a HDC, y la fecha de muerte se registró a partir del servicio de certificados en línea del Registro Civil. La cohorte se dividió en cuatro estratos etarios, en menores de 70 años (grupo 1), de 70 a 74 años (grupo 2), 75 a 79 años (grupo 3) y mayores o igual a 80 años (grupo 4). Se definió como evento principal la muerte del paciente. El fin del seguimiento fue el 21 de abril de 2014.

El análisis estadístico se realizó en el programa STATA versión 12.1. Las variables fueron expresadas en frecuencias, proporciones, medias con desviación estándar, o mediana con rango intercuartílico según su distribución, la cual se verificó mediante la prueba de Komolgorov-Smirnov. Se compararon las variables cualitativas con el test de chi-cuadrado, mientras que las cuantitativas con la prueba de ANOVA, con ajuste de Bonferroni o Kruskall-Wallis en ausencia de distribución normal. Se consideró un valor de $\mathrm{p}<0,05$ como estadísticamente significativo.

El análisis de sobrevida se realizó con curvas de Kaplan-Meier y test de log-rank. Se excluyeron de este análisis los pacientes trasplantados. Se incluyeron curvas alisadas de estimación del riesgo para graficarla probabilidad de mortalidad en el tiempo para los distintos grupos. Se realizó un análisis multivariado con el modelo de riesgo proporcional de Cox para los factores de riesgo de mortalidad general, incluyendo las variables que en la literatura han sido asociadas a mortalidad en esta población de pacientes.

\section{Resultados}

Ingresaron al programa de HDC 459 pacientes durante el período 2010-2012. El seguimiento promedio de la cohorte fue de 27 meses (rango 3 a 52). El grupo 1 correspondió a $65,4 \%$ del total, el grupo 2 a $12,6 \%$, el grupo 3 a $12,4 \%$ y el grupo 4 a 9,8\%. No hubo diferencia en la distribución por sexo entre los grupos, del total de la cohorte 
$55,6 \%$ eran hombres. Se trasplantaron 9 pacientes, todos del grupo 1.

En la Tabla 1 se muestran las características en relación a la causa de ERCT y acceso vascular de la cohorte comparando los grupos etarios. Del total de pacientes $29,8 \%$ correspondían a ERCT por nefropatía diabética, 24,6\% de causa desconocida, $11,5 \%$ de causa glomerular, $9,8 \%$ calificada como nefroesclerosis hipertensiva, $7,6 \%$ por disfunción del injerto renal, $3,7 \%$ por enfermedad poliquística, $3,9 \%$ de origen obstructivo y $8,9 \%$ de causas misceláneas (ocho pacientes por lupus eritematoso sistémico, once por mieloma múltiple, doce por nefritis túbulo-intersticial crónica, seis por nefropatía isquémica, una hipertensión maligna y una necrosis tubular aguda no recuperada).
A mayor edad se observó más pacientes con ERCT de causa desconocida y calificada como nefroesclerosis, con diferencia estadísticamente significativa entre los grupos 1 y 4 para etiología desconocida, y entre el grupo 1 y todos los demás para nefroesclerosis hipertensiva. En el grupo 4 hubo menos nefropatía diabética y una tendencia a menor glomerulonefritis crónica comparado con el grupo 1.

Respecto al acceso vascular no hubo diferencia entre los grupos, del total de los pacientes, $73,4 \%$ inició hemodiálisis con catéter venoso central tunelizado (Tabla 1).

Como se ve en la Tabla 2, la mortalidad al final del seguimiento fue mayor a mayor edad. La mortalidad al año de seguimiento en el grupo de $\geq 80$

Tabla 1. Características basales de la cohorte por grupos etarios, causa de ERCT y acceso vascular

\begin{tabular}{|c|c|c|c|c|c|}
\hline Variable & Grupo 1 & Grupo 2 & Grupo 3 & Grupo 4 & Valor $\mathbf{p}$ \\
\hline Número de pacientes (\%) & $299(65,14)$ & $58(12,64)$ & $57(12,42)$ & $45 \quad(9,80)$ & \\
\hline Hombres (\%) & $165(55,18)$ & $34(58,62)$ & $29(50,87)$ & $27(60)$ & NS \\
\hline $\begin{array}{l}\text { Etiología de ERCT } \\
\text { - Desconocida } \\
\text { - Nefropatía diabética } \\
\text { - Glomerulonefritis crónica } \\
\text { - Enfermedad poliquística } \\
\text { - Nefroesclerosis hipertensiva } \\
\text { - Disfunción crónica del injerto } \\
\text { - Uropatía obstructiva } \\
\text { - Misceláneas }\end{array}$ & $\begin{array}{rr}59 & (19,73) \\
99 & (33,11) \\
44 & (14,71) \\
15 & (5,01) \\
13 & (4,34) \\
33 & (11,03) \\
5 & (1,67) \\
31 & (10,36)\end{array}$ & $\begin{array}{rc}17 & (29,31) \\
16 & (27,58) \\
4 & (6,89) \\
2 & (3,44) \\
8 & (13,79)^{*} \\
2 & (3,44)^{*} \\
5 & (8,62)^{*} \\
4 & (6,89)\end{array}$ & $\begin{array}{rr}17 & (29,82) \\
18 & (31,57) \\
4 & (7,01) \\
0 & \\
11 & (19,29)^{*} \\
0 & \\
3 & (5,26) \\
4 & (7,01)\end{array}$ & $\begin{aligned} 20 & (44,44)^{*} \\
4 & (8,88)^{*+} \\
1 & (2,22) \\
0 & \\
13 & (28,88)^{*} \\
0 & \\
5 & (1,11)^{*} \\
2 & (4,44)\end{aligned}$ & $\begin{array}{c}<0,0001 \\
<0,006 \\
\text { NS } \\
\text { NS } \\
<0,005 \\
0,008 \\
<0,003 \\
\text { NS }\end{array}$ \\
\hline $\begin{array}{l}\text { Acceso vascular } \\
\text { - n de ingresos con catéter (\%) }\end{array}$ & $219(73,24)$ & $45(77,58)$ & $39(68,42)$ & $34(75,55)$ & NS \\
\hline
\end{tabular}

Para comparaciones con más de un valor de p significativo se muestra sólo el mayor. *Significancia estadística respecto al grupo 1. ** Significancia estadística respecto al grupo 2 . + Significancia estadística respecto al grupo 3 . NS $=$ Sin significancia estadística.

Tabla 2. Características basales de la cohorte por grupo etario y comorbilidades

\begin{tabular}{|lcccccc|}
\hline Variables n (\%) & Grupo 1 & Grupo 2 & Grupo 3 & Grupo 4 & Valor p \\
\hline Índice de Charlson & $4,7(3,2)$ & $7(1,4)$ & $7,5(2)$ & $7,9(2)$ & 0,0001 \\
\hline Diabetes Mellitus & $127(42,47)$ & $30(51,72)$ & $23(40,35)$ & $12(26,66)$ & NS \\
\hline Hipertensión arterial & $223(74,58)$ & $50(86,20)$ & $54(94,73)^{*}$ & $41(91,11)$ & 0,001 \\
\hline Enfermedad cardiovascular & $44(14,71)$ & $22(37,93)^{*}$ & $26(45,61)^{*}$ & $22(48,88)^{*}$ & $<0,0001$ \\
\hline Cáncer & $11(3,67)$ & $2(3,44)$ & $6(10,52)$ & $5(11,11)$ & NS \\
Muertes & $66(22,07)$ & $25(43,10)^{*}$ & $32(56,14)^{*}$ & $29(64,44)^{*}$ & 0,001 \\
\hline
\end{tabular}

Para comparaciones con más de un valor de P significativo se muestra sólo el mayor. Índice de Charlson expresado como mediana y rango intercuartílico. Prueba de Kruskal-Wallis para Índice de Charlson. *Significancia estadística respecto al grupo 1. **Significancia estadística respecto al grupo 2. +Significancia estadística respecto al grupo 3. NS = Sin significancia estadística. 

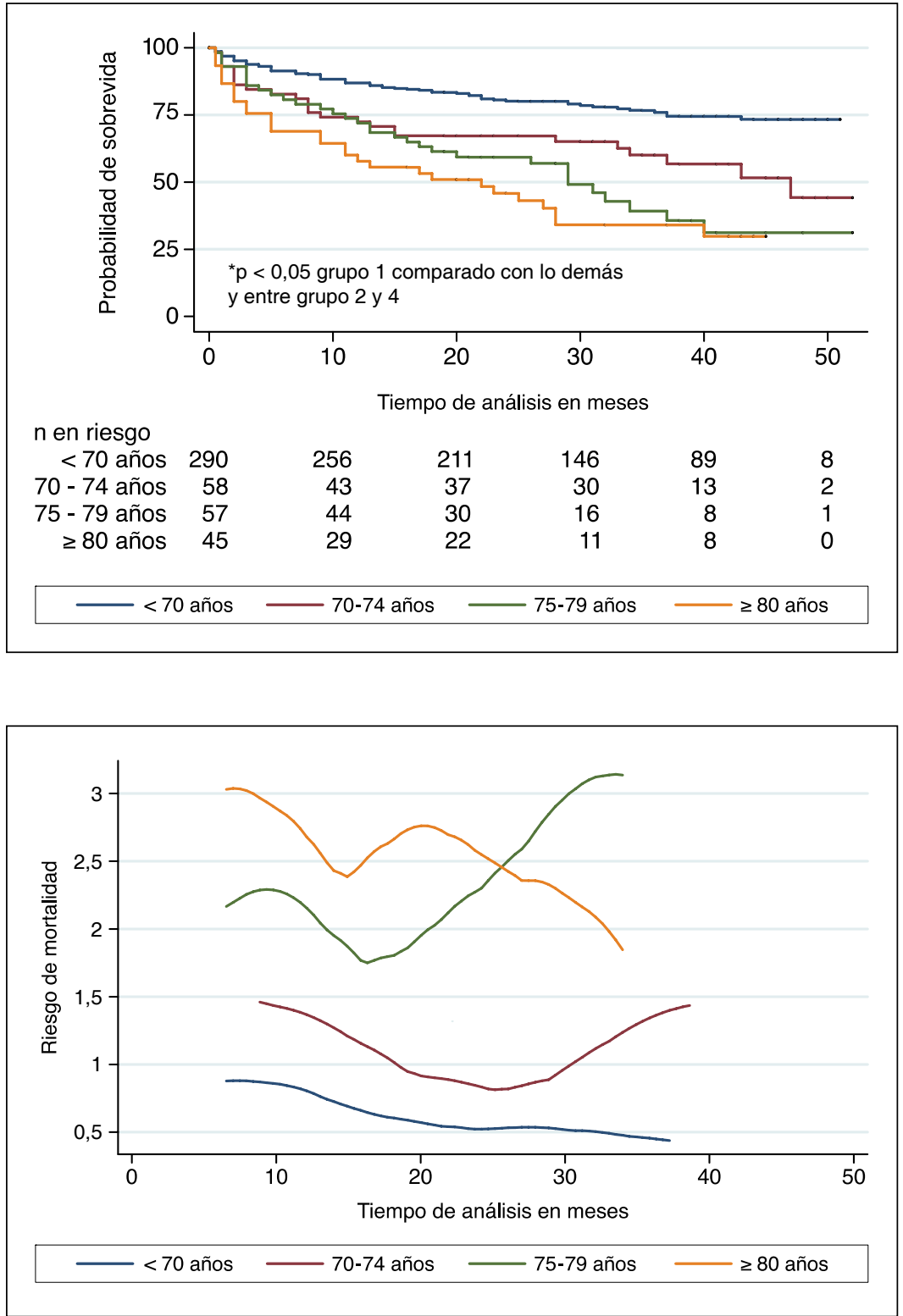

Figura 1. Curvas de sobrevida de Kaplan-Meier y comparación de las tasas de sobrevida con test de log-rank. La mortalidad aumenta con el incremento de la categoría de edad.

Figura 2. Estimación de riesgo de mortalidad en el tiempo. Se observan dos picos de mortalidad en el grupo 3 y 4 , uno a los pocos meses, y el segundo al final del seguimiento para el grupo $3, y$ a los 20 meses para el grupo 4. La forma de la curva sigue un patrón diferente en los grupos más jóvenes, con mortalidad más tardía.

años fue de $43 \%$ mientras que en los $<70$ años, 70-74 años, y 75-79 fue de 13, 26 y 29\% al año, respectivamente. Las curvas de sobrevida (Figura 1) muestran una mejor sobrevida para el grupo 1 , con diferencia significativa respecto a los grupos $2(\mathrm{p}=0,0014)$, grupo 3 y grupo $4(\mathrm{p}<0,0001)$. También hubo una mayor mortalidad del grupo 4 comparado con el grupo $2(\mathrm{p}=0,0175)$.

La estimación de riesgo de mortalidad mediante curvas alisadas, refleja que la probabilidad de morir es mayor en los primeros meses de iniciada la hemodiálisis en los grupos de mayor edad (Figura 2). Para el grupo 4 existe un segundo pico de mortalidad alrededor de los 20 meses, mientras que para el grupo 3 este pico es más tardío. El patrón es diferente para los grupos más jóvenes, en los que una vez iniciada la terapia la probabilidad de morir disminuye durante la fase inicial del seguimiento.

En el análisis con modelo de Cox multivariado 
Tabla 3. Modelo proporcional de riesgo de Cox, análisis multivariado

\begin{tabular}{|c|c|c|c|}
\hline Variable & Hazard Ratio & Intervalo de confianza $95 \%$ & Valor de $p$ \\
\hline Hombre & 1,02 & $0,74-1,41$ & 0,891 \\
\hline 70-74 años & 1,18 & $0,95-1,47$ & 0,127 \\
\hline 75-79 años & 1,37 & $1,15-1,64$ & $<0,05$ \\
\hline$\geq 80$ años & 1,63 & $1,19-2,24$ & $<0,05$ \\
\hline Catéter venoso central & 1,95 & $1,30-2,90$ & $<0,05$ \\
\hline Diabetes mellitus & 0,71 & $0,48-1,04$ & 0,085 \\
\hline Enfermedad cardiovascular & 1,16 & $0,92-1,36$ & 0,205 \\
\hline Cáncer & 0,89 & $0,56-1,41$ & 0,633 \\
\hline Índice de Charlson & 1,45 & $1,38-1,52$ & $<0,05$ \\
\hline
\end{tabular}

Para el Índice de Charlson el modelo supone un aumento de la probabilidad de riesgo por cada unidad de aumento del puntaje.

se observa que la edad mayor a 75 y 80 años, el índice de Charlson y la presencia de catéter venoso central como acceso vascular se asocian de forma significativa a mayor riesgo de mortalidad, con HR de 1.37, 1.63, 1.45 y 1.95 respectivamente (Tabla 3).

\section{Discusión}

En sus inicios la terapia de sustitución renal era considerada sólo en aquellos pacientes jóvenes susceptibles de ser trasplantados, su uso en pacientes de edad avanzada era excepcional. Esto ha cambiado radicalmente en la última década, donde se ha visto un aumento marcado del número de pacientes mayores de 80 años que inician diálisis, tal como ha sido reportado tanto en estudios unicéntricos ${ }^{11}$, como en grandes registros internacionales ${ }^{2-4}$. Por esto es fundamental conocer los resultados de la HDC en este grupo de pacientes y cuáles son los factores que se asocian a peores resultados, de tal manera de llevar a cabo la terapia de la mejor manera y seleccionar a quienes más se benefician de esta.

La mayoría de la información proviene de estudios observacionales. En Francia, en una muestra del registro REIN (2,500 pacientes incidentes de $\geq 75$ años) se vio una mortalidad de $19 \%$ a 6 meses, los factores de riesgo independientes más importantes fueron el inicio de diálisis no planificado, presencia de diabetes, insuficiencia cardíaca, enfermedad vascular periférica, cáncer activo, arritmias, demencia y poca movilidad ${ }^{12}$. En Corea, un análisis de 11.301 pacientes incidentes de $\geq 65$ años, reveló una mortalidad de 30\% el primer año en los octogenarios, con asociación a factores como edad, enfermedad cardiovascular, diabetes, demencia y falta de seguro médico ${ }^{13}$.

En Latinoamérica hay pocos reportes enfocados en la sobrevida del adulto mayor, sin embargo, en los estudios de mortalidad en HDC en adultos en general, los factores asociados a mayor mortalidad siguen siendo la edad, y la presencia de diabetes, tal como lo muestra una numerosa cohorte publicada en Brasil ${ }^{14}$.

Nuestro estudio revela que $34,9 \%$ de los pacientes que inician HDC tienen 70 o más años, y $9,8 \%$ son $\geq 80$ años. Los pacientes de mayor edad presentaron mayor prevalencia de comorbilidades, principalmente cardiovasculares. En el caso de diabetes mellitus, esta se observó en una menor prevalencia en el grupo $\geq$ de 80 años, lo que ha sido también reportado en registros grandes como el DOPPS-III ${ }^{15}$; esto podría deberse a la elevada mortalidad cardiovascular de los pacientes diabéticos a edades más precoces. En relación a las causas de ERCT en este grupo etario los hallazgos son similares a los mencionados en la literatura internacional, menos enfermedad glomerular, poliquistosis, y nefropatía diabética, y más enfermedad hipertensiva, vascular y de causa desconocida ${ }^{3}$.

La mortalidad fue más alta a mayor edad. El grupo de pacientes $\geq 80$ años tuvo una mortalidad al año de $43 \%$, cuatro veces mayor que la pobla- 
ción general de la misma edad en Chile ${ }^{6}$. Llama la atención que $25 \%$ de los sujetos de este grupo falleció en los primeros 3 meses, fenómeno que ha sido ampliamente reportado, y que podría indicar que existe un grupo de pacientes de alto riesgo que no se benefician con la terapia o que requieren consideraciones terapéuticas especiales.

En el análisis multivariado, las variables que se asociaron con mortalidad fueron la edad a partir de los 75 años, el índice de Charlson y el catéter venoso central como acceso vascular. Del total de la muestra 73,4\% inició HDC con catéter, sin diferencia entre los grupos.

Otros estudios también han mostrado mayor mortalidad en pacientes que inician diálisis con catéter versus fístula arteriovenosa (FAV); un registro canadiense de 397.721 pacientes entre los años 2001 y 2010 mostró, en un análisis estratificado por edad, que tener FAV era un factor protector en todos los grupos, con un HR 0,65 para el grupo $\geq 85$ años de edad ${ }^{16}$.

Si bien la presencia de catéter puede asociarse a complicaciones por sí mismo, también es reflejo de falta de control nefrológico al inicio de diálisis no planificada. Esto lo muestra el estudio CHOICE, donde los pacientes que habían sido vistos por nefrólogo un mes previo al inicio de HDC tenían más probabilidad de iniciar diálisis con FAV (39 versus $10 \%)^{17}$.

En nuestro país, el acceso a control nefrológico sigue siendo deficiente pese al desarrollo del plan de Garantías Explícitas en Salud (GES) ${ }^{18}$, esto mismo ocurre en otros aspectos de la terapia de HDC, como el tratamiento de la anemia ${ }^{19}$ y probablemente también el acceso vascular. Esto permite plantear que la falta de acceso vascular definitivo al inicio de HDC puede ser indicador de falta de control nefrológico oportuno previo al inicio del tratamiento.

La tendencia actual apunta al desarrollo de puntajes pronósticos para predecir la mortalidad a 3 meses, de tal manera de introducir algoritmos que permitan derivar a los pacientes añosos a trasplante, hemodiálisis, diálisis peritoneal, o terapia paliativa $^{20}$, si bien esto pudiera ayudar a la toma de decisiones, debe ser ajustado y validado a la realidad local.

Dentro de las fortalezas del estudio está el incluir a pacientes incidentes en HDC de una red de atención que abarca varios centros asistenciales, por lo que es una población heterogénea que parece una muestra representativa de la población urbana de nuestro país. El seguimiento es completo debido a que toda la información está canalizada en el hospital base y en el Registro Civil. Las características de la cohorte muestra hallazgos similares a otras descritas en la literatura, en lo que se refiere a prevalencia de patologías y etiología de la ERC. Como debilidades del estudio está la falta de mayor información respecto a otras características clínicas que pueden influir en el desenlace, como el estado nutricional, la funcionalidad, y los tratamientos farmacológicos recibidos. La falta de información respecto a hospitalizaciones y causas específicas de mortalidad también pueden verse como una limitación, sin embargo, el objetivo del estudio era analizar la mortalidad general.

En suma, en nuestro estudio observamos una mayor mortalidad en el grupo de pacientes de edad avanzada que inician hemodiálisis crónica, en especial en los octogenarios, de ellos $64 \%$ estaban muertos al final del seguimiento, con un pico de mortalidad al inicio de la terapia y a los 20 meses de evolución. Los factores de riesgo asociados a mayor mortalidad, aparte de la edad, fueron la presencia de catéter venoso central como acceso vascular y el índice de Charlson.

Por lo tanto, se puede concluir que la presencia de comorbilidades y un inicio no planificado de HDC afectan negativamente el pronóstico de sobrevida, por lo que es fundamental orientar las políticas de salud a reforzar el control nefrológico oportuno de los pacientes con ERC. Este estudio representa un esfuerzo más en intentar determinar cuáles son las características que pueden predecir una mala evolución de los pacientes de edad avanzada que inician hemodiálisis crónica.

\section{Referencias}

1. Organización Mundial de la Salud. Estadísticas Sanitarias Mundiales 2014. Disponible en: http://www.who. int/research/es/ (Consultado el 14 de diciembre de 2014).

2. Kurella M, Covinsky KE, Collins AJ, Chertow GM. Octogenarians and Nonagenarians Starting Dialysis in the United States. Ann Intern Med 2007; 146: 177-83.

3. U.S. Renal Data System (2014) Annual Data Report: Atlas of Chronic Kidney Disease and End-Stage Renal Disease in the United States. Disponible en: http://www. usrds.org/reference.aspx (Consultado el 14 de diciembre de 2014). 
4. Poblete H. XXXIII Cuenta de Hemodiálisis Crónica en Chile 2013. Sociedad Chilena de Nefrología. Registro de Diálisis. Disponible en: http://www.asodi.cl/ clienteasodi/wordpress/wp-content/uploads/2014/01/ cuenta-final-XXXIII-HDC-2013.pdf (Consultado el 14 de diciembre de 2014).

5. Damianovic N. Población y Sociedad. Aspectos Demográficos 1950-2004. Instituto Nacional de Estadísticas. Chile. Disponible en: http://www.ine.cl/canales/chile_estadistico/familias/demograficas_vitales.php (Consultado el 14 de diciembre de 2014).

6. Encuesta Nacional de Salud 2009-2010. Ministerio de Salud. Chile. Disponible en: http://web.minsal.cl/portal/url/item/bcb03d7bc28b64dfe040010165012d23.pdf (Consultado el 14 de diciembre de 2014).

7. Valdivia G, Domínguez A. Población de 80 años y más en Chile: Una visión preliminar desde el punto de vista epidemiológico. Rev Med Clin Condes 2012; 23 (1): 5-12.

8. Kurella M, Covinsky K, Chertow GM, Yaffe K, Landefeld CS, McCulloch Ch. Functional Status of Elderly Adults before and after Initiation of Dialysis. N Engl J Med 2009; 361:1539-47.

9. Charlson ME, Pompei P, Ales KL, MacKenzie CR. A New Method Of Classifying Prognostic Comorbidity In Longitudinal Studies: Development And Validation. J Chronic Dis 1987; 40 (5): 373-83.

10. Hemmelgarn BR, Manns BJ, Quan H, Ghali WA. Adapting the Charlson Comorbidity Index for use in patients with ESRD. Am J Kidney Dis 2003; 42 (1): 125-32.

11. Sladoje-Martinovic B, Mikolasevic I, Bubic I, Racki S, Orlic L. Survival of chronic hemodialysis patients over 80 years of age. Clin Interv In Aging 2014; 9: 689-96.

12. Cochoud C, Labeeuw M, Moranne O, Allot V, Esnault V, Frimat L, et al. A clinical Score to Predict 6-month Prognosis in Elderly Patients Starting Dialysis for End-Stage Renal Disease. Nephrol Dial Transplant 2009;
24: 1553-61.

13. Lee S, Ryu J-H, Kim H, Kim KH, Ahn HS, Hann HJ, et al. An Assessment of Survival among Korean Elderly Patients Initiating Dialysis: A National Population- Based Study. PLoS ONE 2014; 9 (1): e86776.

14. Campos Szuster D, Teixeira Caiaffa W, Gurgel Andrade E, Acurcio F, Leal Cherchiglia M. Survival Analysis of Dialysis Patients in the Brazilian Unified National Health System. Cad Saúde Pública, Rio de Janeiro. 2012; 28 (3): 415-24.

15. Canaud B, Tong L, Tentori F, Akiba T, Karaboyas A, Gillespie B, et al. Clinical Practices and Outcomes in Elderly Hemodialysis Patients: Results from the Dialysis Outcomes and Practice Patterns Study (DOPPS). Clin J Am Soc Nephrol 2011; 6 (7): 1651-62.

16. Zhang J, Al-Jaishi A, Na Y, de Sa E, Moist L. Association Between Vascular Access Type and Patient Mortality Among Elderly Patients on Hemodialysis in Canada. Hemodial Int 2014; 18: 616-24.

17. Astor BC, Eustace JA, Powe NR, Klag MJ, Sadler JH, Fink NE, et al. Timing Of Nephrologist Referral And Arteriovenous Access Use: The CHOICE Study. Am J Kidney Dis 2001; 38 (3): 494-501.

18. González F. La implementación del Plan de Acceso Universal y Garantías Explícitas (Plan AUGE) ha deteriorado la calidad del tratamiento de pacientes con insuficiencia renal terminal. Rev Med Chile 2006; 134: 1288-94.

19. González F. El tratamiento del plan AUGE (GES) de la anemia en diálisis crónica no ha sido eficaz y, probablemente, no se han aprovechado los recursos económicos destinados para ello. Rev Med Chile 2006; 134: 1288-94.

20. Couchoud CG, Beuscart JB, Aldigier JC, Brunet P, Moranne OP. Development of a risk stratification algorithm to improve patient-centered care and decision making for incident elderly patients with end-stage renal disease. Kidney Int 2015; 88: 1178-86. 